\title{
Design and performance studies of micromegas chambers for the ATLAS muon spectrometer upgrade
}

\author{
Konstantinos Ntekas \\ National Technical University of Athens, Brookhaven National Laboratory \\ Email: konstantinos.ntekas@cern.ch \\ on behalf of the ATLAS Muon Collaboration
}

\begin{abstract}
Micromegas, an abbreviation for Micro MEsh Gaseous Structure (MM), is a robust detector with excellent spatial resolution and high rate capability. An $R \& D$ activity, called Muon ATLAS MicroMegas Activity (MAMMA), was initiated in 2007 in order to explore the potential of the MM technology for use in the ATLAS experiment. After several years of prototyping and testing, the ATLAS collaboration has chosen the micromegas technology along with the small-strip Thin Gap Chambers (sTGC) for the upgrade of the inner muon station in the high-rapidity region, the so called New Small Wheel (NSW) upgrade project. It employs eight layers of MM and eight layers of sTGC detectors. The NSW project requires fully efficient micromegas chambers, able to cope with the maximum expected rate of $15 \mathrm{kHz} / \mathrm{cm}^{2}$ featuring spatial resolution better than $100 \mu \mathrm{m}$. The MM detectors will cover a total active area of $\sim 1200 \mathrm{~m}^{2}$ and will be operated in a moderate magnetic field with intensity up to $0.4 \mathrm{~T}$. The required track reconstruction accuracy is provided by the intrinsic detector space resolution, accompanied by a mechanical precision at the level of $30 \mu \mathrm{m}$ along the precision coordinate. Moreover, together with the precise tracking capability the NSW MM chambers will contribute to the ATLAS Level-1 trigger system. An extensive R\&D program is ongoing to determine the best configuration that satisfies these requirements. Several tests have been performed on small $\left(10 \times 10 \mathrm{~cm}^{2}\right)$ and medium $\left(1 \times 1 \mathrm{~m}^{2}\right)$ size prototypes, including also the recently developed MM quadruplet prototype, using medium $(1-5 \mathrm{GeV} / \mathrm{c})$ and high momentum $(120-150 \mathrm{GeV} / \mathrm{c})$ hadron beams at CERN. A brief overview of the results obtained from recent design and performance tests concerning the aspects discussed above is presented.
\end{abstract}

\section{INTRODUCTION}

The upgrade of the Large Hadron Collider (LHC) at CERN foresees a luminosity increase by a factor 5. To cope with the corresponding rate increase, the ATLAS detector [1] needs to be upgraded. The upgrade will proceed in two steps: Phase-I in the LHC shutdown 2018/19 [2] and Phase-II in 2023-25 [3].

\section{A. The ATLAS New Small Wheel upgrade project}

The largest of the ATLAS Phase-I upgrades concerns the replacement of the two first muon stations, called Small Wheels (SW), in the high-rapidity region with New Small Wheels [4]. The NSW is expected to cope with the highest rates expected in Phase-II and enhances the performance of the forward muon system by adding trigger functionality to the first muon station.

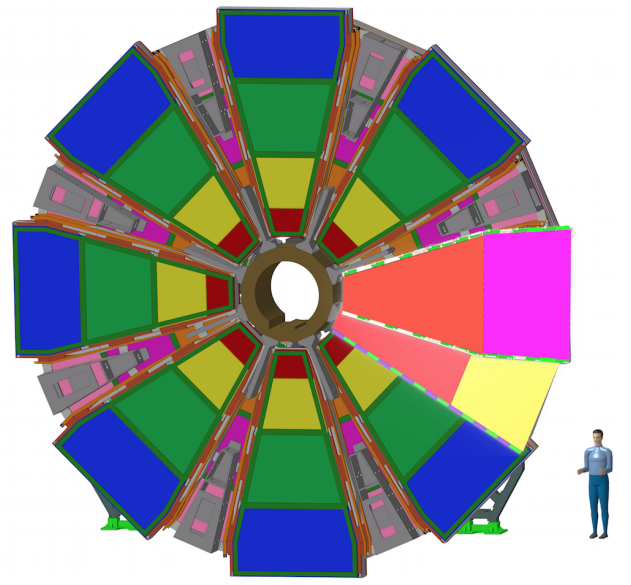

Fig. 1: Graphical representation of an assembled NSW. Two micromegas sectors, one small and one large, are also shown. In reality the MM will not be visible sitting in between the 2 sTGC quadruplets as shown in Fig. 2

The NSW employs eight layers of micromegas detectors (MM) and eight layers of small-strip Thin Gap Chambers (sTGC) [5]. Both technologies will provide tracking and triggering information, resulting into a fully redundant system. Each of the two wheels will consist of sixteen sectors divided into 8 large, 8 small sectors according to their size. A graphical representation of one NSW is shown in Fig. 1. The two technologies will be combined in four wedges per sector following the sTGC-MM-MM-sTGC configuration as shown in Fig. 2. One wedge is basically a quadruplet combining four detecting layers of the same technology.

There will be four types of MM quadruplets in the NSW distributed in small and large sectors: SM1 (lower radius) and SM2 (larger radius) for the small sectors and LM1, LM2 respectively for the large sectors (Fig. 2). All quadruplets have trapezoidal shapes with dimensions between $2-3 \mathrm{~m}^{2}$. Each quadruplet combines two layers with readout strips parallel to the trapezoid bases, measuring the precision coordinate (pseudorapidity $\eta$ ), and two layers with stereo strips, tilted by $\pm 1.5^{\circ}$ with respect to the $\eta$ strips, allowing for the $2^{\text {nd }}$ coordinate (azimuthal) measurement. The strip pitch will be 425 and $450 \mu \mathrm{m}$ for the small and the large sectors respec- 
tively, resulting in $\sim 2 \mathrm{M}$ readout channels in total for the MM system of the NSW.

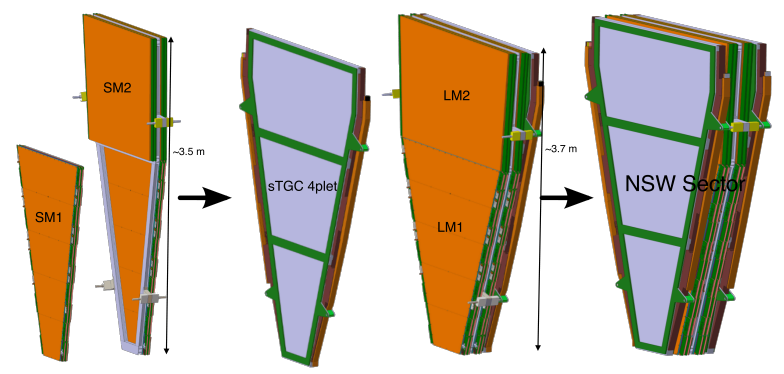

Fig. 2: Combining the four wedges (quadruplets) into a NSW sector. The dimensions of MM small and large sectors accordingly along with the four different types of MM modules (SM1, SM2, LM1, LM2) are shown.

\section{DESIGN STUdies OF MM DETECTORS}

The micromegas technology was developed in the middle of the 1990's introducing a micropattern gaseous detector with two asymmetric regions [6]. Standard MM detectors consist of a planar (drift) electrode, a gas gap of a few millimeters thickness acting as conversion and drift region, and a thin metallic mesh placed at $\sim 100-150 \mu \mathrm{m}$ distance from the readout electrode, defining the amplification region. A 3D design of the MM structure is shown in Fig. 3 (left). The $\mathrm{HV}$ values are chosen such that the electric field in the drift region is a few hundred $\mathrm{V} / \mathrm{cm}$, and $40-50 \mathrm{kV} / \mathrm{cm}$ in the amplification region achieving gas gain values of the order of $10^{4}$ for the standard $\mathrm{Ar}+7 \% \mathrm{CO}_{2}$ gas mixture. The large multiplication factor is essential for the detection of minimum ionizing particles with high efficiency.
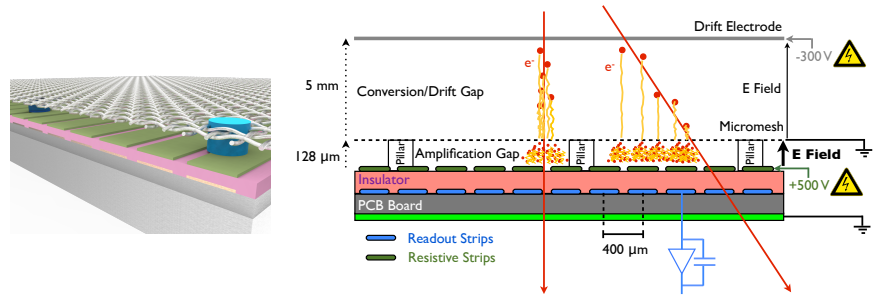

Fig. 3: Left: A 3D schematic of the resistive MM's mesh and readout structure. The mesh is positioned on top of the pillars which define the size of the amplification region. A layer of resistive strips is put above the copper readout strips following the same pattern. Right: Cross section of the MM structure (not at scale). The various detector elements along with the working principle of the detector are illustrated. The dimensions and $\mathrm{HV}$ values noted refer to the majority of the small prototype chambers that have been used at the test beams.

Charged particles traversing the drift space ionize the gas and the electrons, liberated by the ionization process, drift towards the mesh (in tens of nanoseconds). With an electric field in the amplification region $\sim 50-100$ times stronger than the drift field, the mesh is transparent to more than $95 \%$ of the electrons. The electron avalanche takes place in the thin amplification region in less than a nanosecond, resulting in a fast pulse on the readout strip [7]. The drifting of the electrons and the avalanche formation in the amplification region for perpendicular and inclined, with respect to the chamber plane, tracks is illustrated in Fig. 3 (right). The ions that are produced during the avalanche process move towards the mesh with velocities about 100 times smaller than the electrons.

In the case of highly ionizing particles the Raether limit may be reached and discharges (sparks) start to occur. Sparks may damage the detector structure or the readout electronics and lead to large dead times as a result of high voltage breakdown. By adding a layer of resistive strips on top of a thin insulator directly above the readout electrode, as shown in the illustrations of Fig. 3, the MM becomes spark-insensitive. The readout electrode is no longer directly exposed to the charge created in the amplification region. The signal on the readout strip is partly induced from the motion of the ionization charges and partly capacitively coupled to it from the resistive strips. By adding the resistive protection some fraction of the signal height is lost but the chamber can be operated at higher gas gain because sparking is reduced by about three orders of magnitude while the electric field distortion is constrained in a small region of the detector [8].

Hence, resistive MMs can be efficiently operated in a highrate environment as was demonstrated by testing small resistive prototypes inside the ATLAS experiment with particle rates up to $80 \mathrm{kHz} / \mathrm{cm}^{2}$ [10], [11].

\section{A. Construction of large modules}

With the sparking issue solved, the next challenge was to move towards the construction of large area MM. The NSW project will require large MM modules to be built with individual surfaces up to $\sim 3.1 \mathrm{~m}^{2}$ covering a total area of $\sim 1200 \mathrm{~m}^{2}$. In order to satisfy the performance criteria of the ATLAS experiment the position of any NSW chamber element should be known with an uncertainty smaller than $30 \mu \mathrm{m}$ and $80 \mu \mathrm{m}$ for the precision coordinate and the coordinate perpendicular to the precision one respectively. These requirements imply strict mechanical precision during the production and the assembly of the NSW modules.
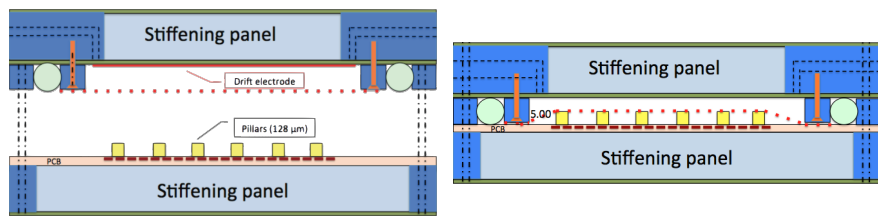

Fig. 4: Sketch of the MM assembly procedure with the floating mesh technique. The stretched mesh is integrated in the drift panel (left) and is then positioned on top of the pillars (right).

One of the issues to be resolved moving towards large size modules, was the limitation in the dimensions imposed by the bulk technique [12] of PCB manufacturing $(\leq 60 \mathrm{~cm})$ which was used so far. While in the bulk MM the metallic mesh is integrated into the readout PCB structure, a new scheme, called "floating mesh technique", was proposed enabling the construction of large area MM. The mesh is integrated in the drift panel containing the cathode plane as shown in Fig. 4 (left). Thus, the drift gap which is separated from 
the readout plane no longer depends on the PCB dimensions. The mesh is stretched and then glued on the aluminum frame surrounding the drift stiffening panel. The mesh support pillars are deposited on top of the resistive strips of the readout panel, defining the size of the amplification gap. Then the integrated drift panel is precisely coupled to the readout panel by positioning the mesh on top of the pillars as shown in Fig. 4 (right).
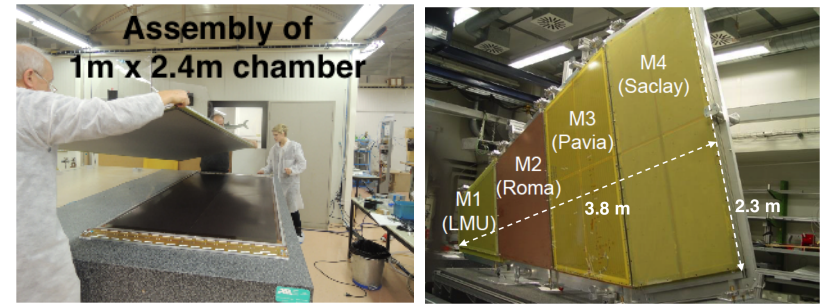

Fig. 5: Left: Photo of one of the large MM functional prototypes during the assembly procedure [9]. Right: Photo of the mechanical MM prototypes, that were built in four different sites, mounted on a frame for mechanical deformation measurements.

Before starting the construction of the final MM prototypes (Module-0), several large size single plane functional MM prototypes (up to $2.4 \mathrm{~m}^{2}$ ) have been built and tested at CERN. A photo from the assembly procedure of such a prototype is shown in Fig. 5 (left). Moreover, in order to establish the design and the construction procedures for the NSW MM chambers, detailed studies of panels construction including extensive prototyping and tests are necessary. Along these lines, large mechanical prototypes have been built in different construction sites following different assembly procedures. The aim of these prototypes, shown on Fig. 5 (right), was mainly to test the different construction/assembly approaches but it also allowed the construction groups to gain valuable experience with building large MM modules.

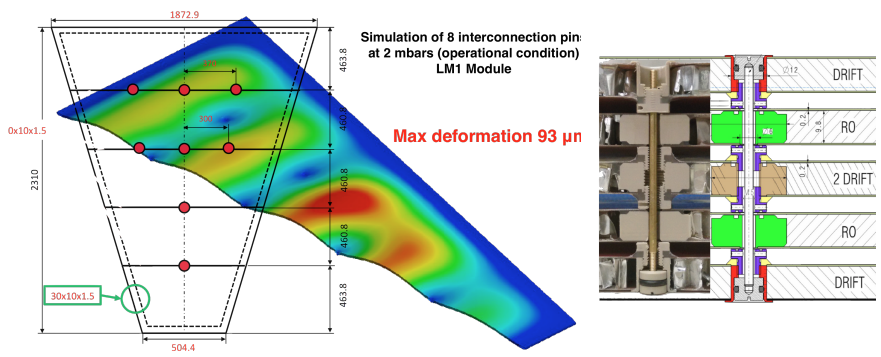

Fig. 6: Left: An example of mechanical simulation of the interconnection scheme regarding the LM1 MM module. The scheme illustrated confines the maximum deformation, due to 2 mbar gas over pressure, to $93 \mu \mathrm{m}$. Right: Photo and sketch of one mechanical prototype of the interconnection mechanism, passing a screw through all the layers of the module.

In parallel, a campaign of measurements of deformations accompanied with mechanical simulations models has evolved. One of the critical issues that were put under study was the effect of gas over pressure on large area modules. The drift panel planarity of a large single gap prototype, with
3 mbar gas over pressure, has been measured observing a maximum deformation of $300 \mu \mathrm{m}$. A series of simulation studies were then performed assessing possible solutions to the observed significant deformation. The collaboration is converging towards a scheme of interconnections along the surface of the large modules, guiding screws across the 4 gaps of the quadruplet through precisely drilled holes. The number and the positions of the interconnections is optimized though simulations (Fig. 6 (left)) while mechanical prototypes of the interconnection scheme have also been realized and tested (Fig. 6 (right))

\section{B. Design \& construction of an ATLAS-like quadruplet proto- type}

A step forward in the prototype production, towards establishing the construction and assembly procedures for the NSW MM modules, was made in the first half of 2014 with the construction of the first MM quadruplet (MMSW). This prototype features a configuration similar to the one foreseen for the NSW MM quadruplets. A cross section schematic of the quadruplet's internal structure is shown in Fig. 7 (left). The prototype is shown in the photo of Fig. 7 (right).
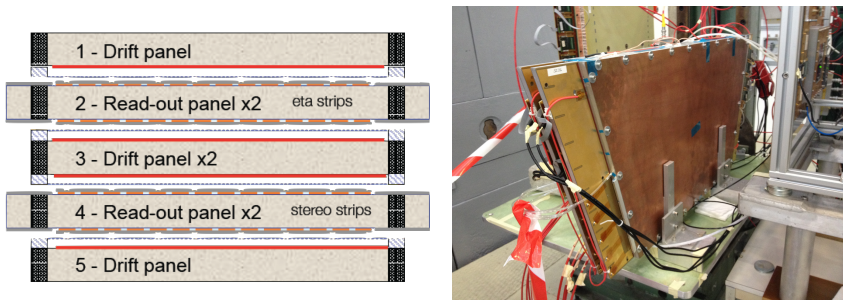

Fig. 7: Left: Cross section of the MMSW prototype internal structure featuring 2 double sided readout panels, 1 double sided and 2 single sided drift panels. Right: A photo of the MMSW prototype installed in the experimental area at CERN during August 2014 test beam.

The readout planes are disposed in a "back-to-back" configuration on two stiffening panels with an aluminum honeycomb internal structure (readout panels). One of them hosts two layers of strips measuring the precision coordinate while the two readout layers of the second panel have stereo strips tilted by $\pm 1.5^{\circ}$ with respect to the precision ones, similar to the configuration described in section I. One central, double sided panel and two external panels sustaining the stainless steel mesh (drift panels) are then coupled to the two readout panels to form the four gas gaps of the quadruplet. The detector has been realized using the resistive-strip technology and decoupling the mesh from the readout structure (floating mesh technique). The four readout planes are segmented into strips with a pitch of $415 \mu \mathrm{m}$ comprising a total of 4096 strips.

\section{PERFormance STUdies OF MiCROMEgAS PROTOTYPES}

In order to demonstrate the performance characteristics of the MM technology and also to optimize the design and operational characteristics of the detector, several prototypes have been tested with high energy particle beams. The studies included testing of chambers of various sizes with different 
characteristics in different beam environments. Moreover due the fact the MM will have to cope with magnetic field intensities up to $0.4 \mathrm{~T}$ in the NSW, dedicated test setups, placing the chambers in a magnet, at CERN and in DESY, were used. For the results that are presented here the front-end electronics were based on the APV25 ASIC (128 channels) [13] readout through the Scalable Readout System (SRS) developed by the RD51 collaboration [14]. The APV25 will not be employed in the NSW. The front-end ASIC providing the trigger and tracking primitives for both the MM and sTGC of the NSW will be the VMM [15] currently being produced in its second prototype version.
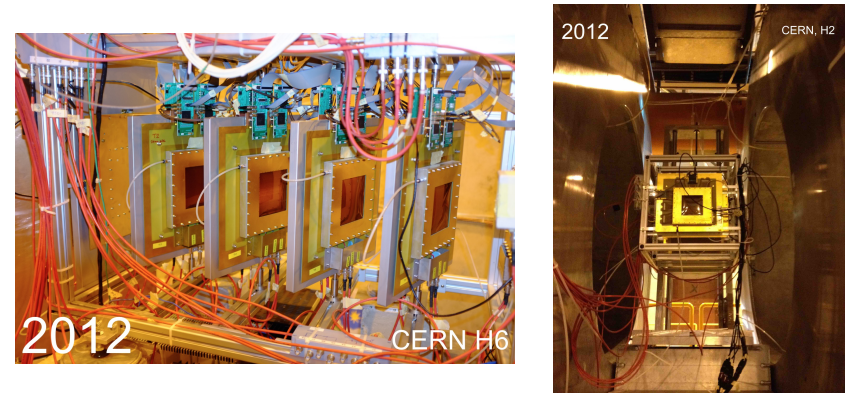

Fig. 8: Photos from test beam periods at CERN North Area (H6, H2 respectively) during the summer of 2012. A telescope of 8 small $\left(10 \mathrm{~cm}^{2}\right)$ prototypes were exposed to a high momentum $(120-150 \mathrm{GeV} / \mathrm{c}) \pi^{+}$beam (left). The chambers were also put inside a magnet to test their performance under the influence of a magnetic field (right).

\section{A. Hit \& track reconstruction techniques}

One of the most attractive characteristics of the MM technology is its excellent spatial resolution due to the fine readout segmentation. Also, because of the asymmetric field configuration and the large multiplication in the amplification region, the chambers are sensitive to a single primary ionization electron. By analyzing the induced pulse on each strip the arrival time of each electron is measured, allowing the detector to work as TPC allowing for precise reconstruction of track segments with a single detector plane. Using the recorded charge and time information, two different hit reconstruction techniques have been developed:

- (Charge) Centroid

Average of the strip position weighted by the charge.

- $\quad \mu \mathrm{TPC}$

2D track reconstruction transforming the arrival time to distance. The hit is defined as the interpolation of the track in the middle of the drift gap.

The charge centroid method reconstructs hits with high accuracy when the particle traverses the chamber plane perpendicularly. In this case the charge is shared among 2-3 strips of one cluster allowing for a very precise charge interpolation. The spatial resolution determined with this method is well below $100 \mu \mathrm{m}$ for perpendicular tracks. The $\mu \mathrm{TPC}$ method does not provide accurate results in this case as the pulse in each strip is the aggregation of pulses induced by more than one electrons. When the particle crosses the detector under an angle the signal induced in each strip is most probably coming from one primary electron with several strips fired from a single track. In this occasion the $\mu \mathrm{TPC}$ method provides a very accurate measurement of the particle hit position on the detector. The charge centroid method accuracy decreases rapidly at larger incident angles, because the charge is distributed along several strips.

Moreover an algorithm combining the hits reconstructed with the two different methods has been developed achieving single plane hit reconstruction with a resolution better than $100 \mu \mathrm{m}$ independently of the track incident angle [16].

\section{B. Refinement of the $\mu T P C$ method}

The performance of the $\mu$ TPC track reconstruction method is of great importance for the Micromegas community and also for the ATLAS NSW upgrade project where the particle track angles expected are between 8 and $30^{\circ}$. Thus, a lot of effort has been dedicated in optimizing this technique.
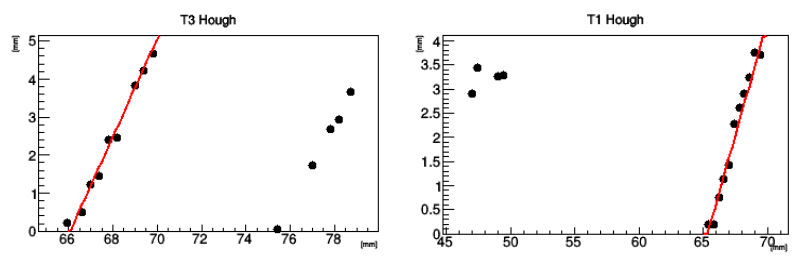

Fig. 9: Displays for a double track (left) event and an event with noise (right) reconstructed in the drift gap of a single chamber. With the red line the track that is reconstructed using the Hough transform technique is shown. Only the hits that belong to the Hough track are then used for reconstructing the single plane segment.

One of the issues that were studied was the refinement of the clustering algorithm to properly treat events with more than one track, or events with noise and/or delta rays ( $\sim 10 \%$ of the events). In these cases, instead of combining all the strips with a signal into one cluster, a more refined algorithm is needed that will be able to identify the real tracks and filter out the noisy strips (Fig. 9) and the strips fired from delta ray. A pattern recognition technique, using the Hough transform, has been developed which filters out the real tracks increasing the efficiency of the event selection and improving the accuracy of the reconstructed track by removing outlying strips [17].

Moreover, due to the readout/resistive elements geometry, there is a non-negligible capacitance between the strips causing the ionization charge to be shared among the neighboring strips. The effect has been extensively studied in simulation concluding that $\sim 10 \%$ of the charge induced on a strip is capacitively coupled to its neighbors ${ }^{1}$. This may bias the reconstructed track by artificially increasing the cluster size in cases where the strips on the edges of the cluster have signal coming only from the coupling with their neighbors. A filtering algorithm has been developed identifying such hits and discarding them from the track reconstruction procedure.

\footnotetext{
${ }^{1}$ The effect depends on the strip pitch and width. For this study a geometry with strips $300 \mu \mathrm{m}$ wide separated by a gap of $100 \mu \mathrm{m}$ was used.
} 
Another parameter that should be treated carefully is the assignment of the hit position with respect to the strip pitch. Due to the finite size of the readout elements, the knowledge of the hit position is limited by the strip pitch value. In the offline reconstruction each hit position is usually assigned in the middle of the strip pitch. In fact, this is correct only for the middle strips of the cluster where the hit position probability is uniformly distributed along the strip pitch. For the edges of the cluster it is more probable for the hit position to be towards the strip pitch edge neighboring to the cluster strips. The assignment of the hit in the middle of the strip pitch for the cluster edges results in biasing the reconstructed track towards larger inclination values, thus a method has been developed tuning the hit position on the cluster edges based on their charge.
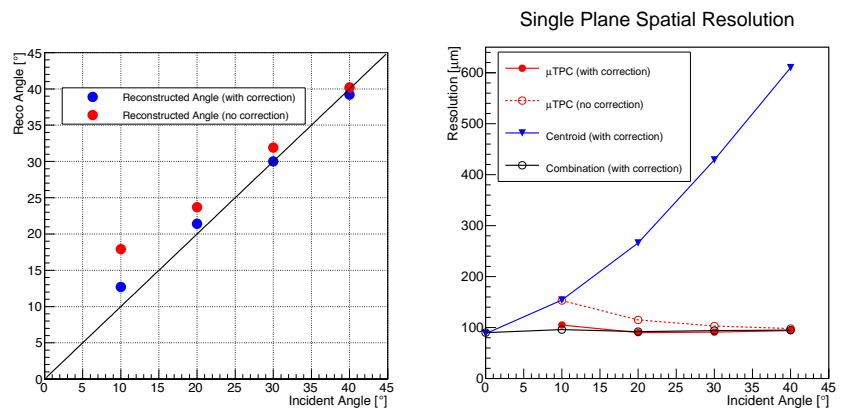

Fig. 10: Left: The reconstructed incident track angle, peak value of the angular distribution, versus the true inclination angle before (red points) and after the correction (blue points). Right: The spatial resolution along the different incident angles measured before and after the correction with the $\mu \mathrm{TPC}$ (red circles) and the centroid (blue triangles) methods. The resolution measured when combining the two methods, after the correction, is also shown (black circles).

The refined $\mu \mathrm{TPC}$ recipe was applied in data taken during July 2012 test beam at CERN where small size $\left(10 \times 10 \mathrm{~cm}^{2}\right)$ prototypes were exposed to a $120 \mathrm{GeV} / \mathrm{c} \pi^{+}$beam and results with and without the new recipe are compared. The improvement in the accuracy of the $\mu \mathrm{TPC}$ method, using the techniques described above, is not only evident in the better agreement of the measured incident angle with the true one shown in Fig. 10 (left), but also in the measurement of the spatial resolution which is displayed in Fig. 10 (right). The impact is more significant for smaller incident angle values.

\section{Performance studies inside a magnetic field}

In the presence of a magnetic field perpendicular to the electric field direction the ionization electrons are no longer drifting along the electric field lines but they follow a direction determined by the Lorentz angle. The total drift velocity consists in this case of two components, one along the drift electric field and one along the $\mathbf{E} \times \mathbf{B}$ direction. A Garfield [18] simulation of the drfit of the electrons inside the conversion region under the influence of the magnetic field for two different magnetic field orientations is shown in Fig. 11.

In order to study the performance of MM chambers under the influence of a magnetic field, the data obtained from a
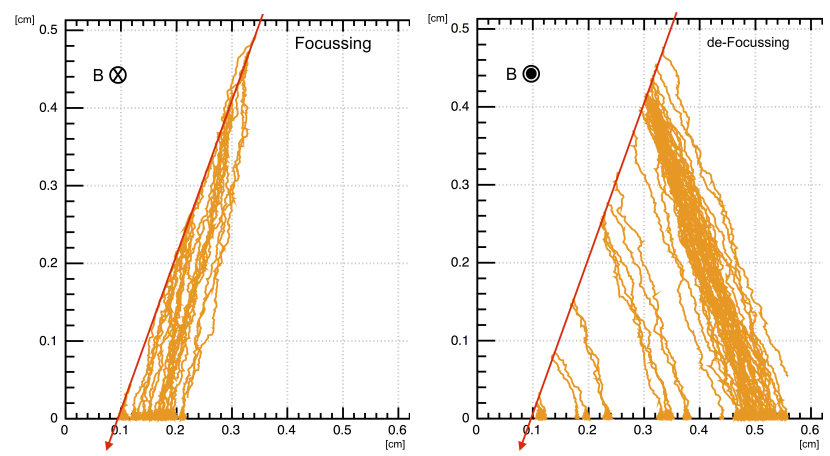

Fig. 11: Simulation of a muon traversing the detector volume under an angle and under the influence of a magnetic field perpendicular to the electric field. Depending on the magnetic field orientation the size of the track footprint can be constricted (left) or expanded (right).

dedicated test beam at H2 CERN north area were used. A set of 4 small size prototypes were installed inside a superconducting magnet that could provide magnetic field with an intensity up to $2 \mathrm{~T}$. The setup was exposed to a $150 \mathrm{GeV} / \mathrm{c} \pi^{+}$beam and was orientated in such a way that the magnetic field was always perpendicular to the electric field direction.
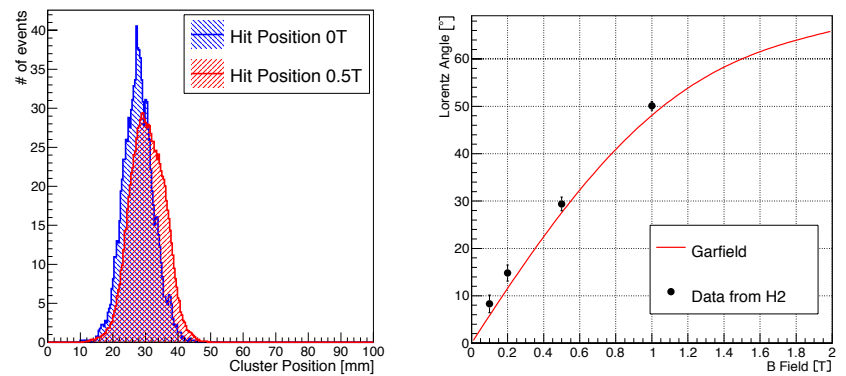

Fig. 12: Left: The beam profiles, using the reconstructed cluster position, for a run without magnetic field (blue distribution) and a run with $0.5 \mathrm{~T}$ magnetic field (red distribution) are shown. Right: The Lorentz angle, calculated from the movement of the beam profile, as a function of the magnetic field intensity (black points). The measurements show very good agreement with the Lorenz angle values expected from Garfield simulation (red line).

When the ionization charges are deflected, due to the effect of the magnetic field, a displacement of the beam profile with respect to the zero magnetic field case is expected. Fig. 12 (left) shows the beam profiles on one chamber for different magnetic field intensities. By measuring the displacement and using the chamber's drift gap dimensions ${ }^{2}$ we calculate the Lorentz angle for the different field values which is then compared to the theoretical values of the Lorentz angle expected from Garfield simulations Fig. 12 (right).

When inclined with respect to the beam axis, the MM detector is able to work as a TPC ( $\mu$ TPC). In Figure 13

\footnotetext{
${ }^{2}$ The drift gap is usually constructed with a $5-10 \mu \mathrm{m}$ accuracy.
} 
(left) the distribution of the drift times recorded using one chamber inclined by $20^{\circ}$ is plotted for runs taken under different magnetic field intensities $(0.5,1 \mathrm{~T})$ during the $\mathrm{H} 2$ test beam. The width of the distribution becomes larger with increasing field as expected due to spreading of the ionization charges. By fitting the full time distribution the width of this spectrum is acquired. This value is indicative of the maximum recorded drift time in the respective chamber's gas gap which for this specific example has a size of $5 \mathrm{~mm}$. Using the measured maximum drift time the drift velocity of the gas can be measured. The measurement was performed for magnetic field intensities $0.2,0.5$ and $1.0 \mathrm{~T}$. The results, shown in Fig. 13 (right) display very good agreement with theoretical values from Garfield simulation [19].
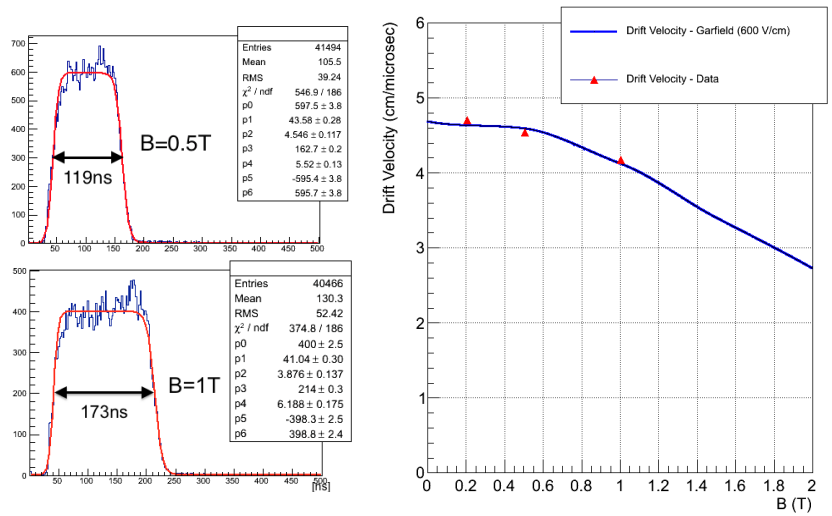

Fig. 13: Left: Drift time distributions recorded with a chamber inclined by $20^{\circ}$ for $0.5 \mathrm{~T}$ (top) and $1 \mathrm{~T}$ (bottom) magnetic field intensity. The width of the time distribution is equal to the maximum recorded drift time and can be used to measure the gas drift velocity. The chamber's drift gap is $5 \mathrm{~mm}$ wide. Right: The drift velocity values measured for the different magnetic field intensities (red triangles) compared to the theoretical expectations from Garfield simulations.

\section{Performance studies of the ATLAS type quadruplet proto- type}

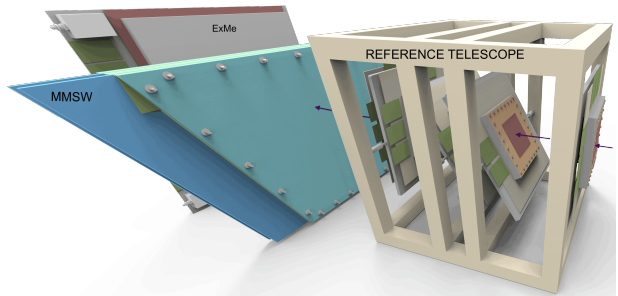

Fig. 14: 3D schematic of the experimental setup used for testing the MMSW prototype. A telescope with four small MM prototypes was used to provide a reference track for the MMSW performance studies.

The performance of the recently developed ATLAS type quadruplet (MMSW) was studied in a dedicated test beam at CERN using $\pi, p$ beam of $6-9 \mathrm{GeV} / \mathrm{c}$. The MMSW was positioned in the beam along with a telescope equipped with small prototype $\mathrm{MM}$ chambers providing the $2 \mathrm{D}$ reference track. The MMSW's operation, using the standard $\mathrm{Ar}+7 \% \mathrm{CO}_{2}$ gas mixture, was smooth during the whole period of irradiation with all the four layers being more than $99 \%$ efficient. For the preliminary results presented here the chamber was kept perpendicular to the beam axis.
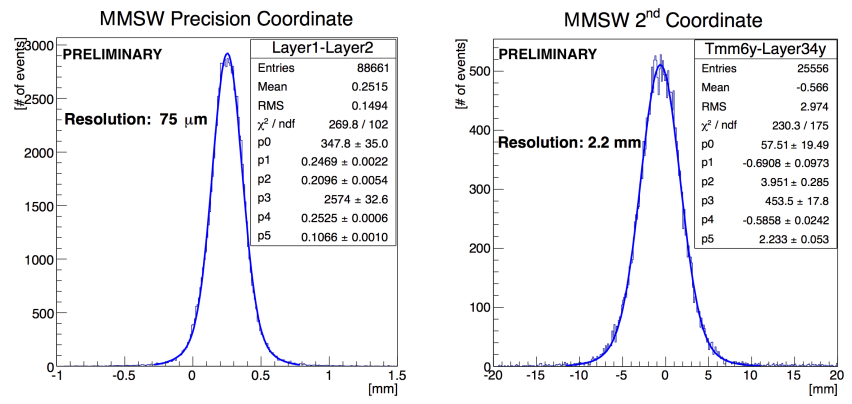

Fig. 15: Left: Residual distribution of the reconstructed hit positions in the precision coordinate layers of the MMSW prototype. Right: Residual distribution of the MMSW second coordinate hit position with a second coordinate hit coming from one of the reference chambers. The distributions are fitted with double gaussian functions to take also into account the tails. For the left plot the width of the core gaussian is divided by $\sqrt{2}$, assuming equal spatial resolution of the two layers, giving an estimation of the MMSW spatial resolution for the precision coordinate. The distribution is not centered at zero due to a small rotation $\left(<1^{\circ}\right)$ of the chamber with respect to the beam axis.

By plotting the hit position difference between the first two layers of the MMSW, both with readout strips measuring the precision coordinate, we were able to determine the spatial resolution of each of the 2 precision layers equal to $75 \mu \mathrm{m}$ as shown in Fig. 15 (left). This value is in accordance with the measurements performed in the past, using small prototypes with $400 \mu \mathrm{m}$ readout strip pitch, determining a resolution in the order of $70 \mu \mathrm{m}$, taking into account the slightly larger strip pitch in the quadruplet module $(415 \mu \mathrm{m})$.

Combining the two stereo strip layers of the quadruplet the second coordinate can also be reconstructed. Simulation studies of the stereo strips configuration dictate, that by combining two stereo layers, with a $3^{\circ}$ angle between them, as shown in Fig. 16 (left), the second coordinate can be reconstructed with an uncertainty of $\sim 2.2 \mathrm{~mm}$, assuming single layer spatial resolution of $\sim 80 \mu \mathrm{m}$ [19].

The chambers of the reference telescope are used to reconstruct the second coordinate of the extrapolated track on the MMSW frame. By comparing this with the second coordinate hit reconstructed combining the two stereo readout information the spatial resolution of the second coordinate is measured to be of the level of $2.2 \mathrm{~mm}$ (Fig. 15). This measurement is slightly worse than what we expect from simulation, looking at the blue curve of Fig. 16 (right), because it includes also the extrapolation error due to the distance between the telescope and the MMSW. Also an additional contribution to the uncertainty measured due to multiple scattering should be taken into account. 


\section{CONCLUSIONS}

After an intensive R\&D phase, which begun in 2007, the MM technology has evolved significantly over the last years and it was finally approved as one of the technologies that will be used in the NSW upgrade project of the ATLAS experiment. This is the first application of a Micropattern Gaseous Detector (MPGD) technology in a very large scale experiment and in a high rate environment.

Recently the efforts have been aimed towards establishing the construction and assembly procedures ensuring the construction of large MM detectors with very good mechanical precision. A series of large mechanical and functional prototypes have been produced and tested accompanied by extensive measurements and simulation studies in order to converge to the most appropriate design and layout for the NSW detectors. In parallel a series of performance studies is ongoing in order to optimize the detector performance and operating conditions taking also into account that the detectors of the NSW will have to operate inside a magnetic field with intensity up to $0.4 \mathrm{~T}$.
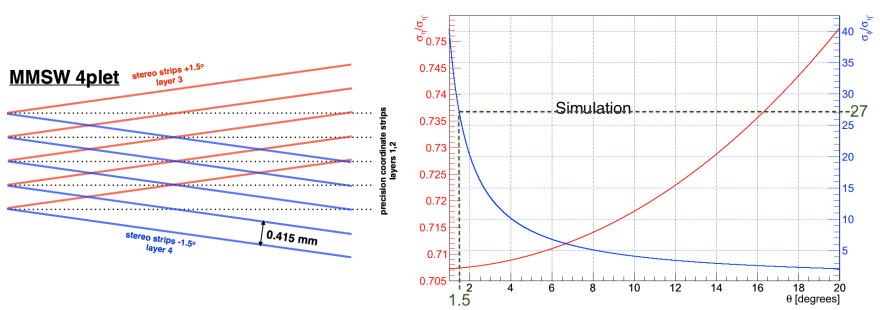

Fig. 16: Left: Schematic of the readout strip orientation for the four layers of the MMSW prototype. The two stereo strip readouts (red, blue lines) are tilted by $\pm 1.5^{\circ}$ with respect to the two precision strip readouts (black lines). The strip pitch is $415 \mu \mathrm{m}$ for all the four layers. Right: The spatial resolution of the second coordinate $\sigma_{\phi^{\prime}}$, reconstructed using two stereo systems with spatial resolution $\sigma_{\eta^{\prime}}$ and tilted by an angle $2 \theta$ between each other, divided by $\sigma_{\eta^{\prime}}$ as a function of the angle $\theta$ (blue curve). The red curve shows the ratio between the spatial resolution of he precision coordinate $\left(\sigma_{\eta^{\prime}}\right)$ that is reconstructed using the two stereo layers and the single layer spatial resolution $\left(\sigma_{\eta}\right)$ as a function of the angle $\theta$.

Moreover, the first MM quadruplet prototype has been produced in the summer of 2014. The so-called MMSW prototype follows the layout principle of the MM quadruplets that will be built for the NSW and preliminary results from recent performance tests are very encouraging. The studies presented are essential for the MM detectors of the NSW project which is now entering the production phase with final full size prototypes along with new readout electronics expected to be built in 2015 .

\section{ACKNOWLEDGMENT}

The present work was co-funded by the European Union (European Social Fund ESF) and Greek national funds through the Operational Program "Education and Lifelong Learning" of the National Strategic Reference Framework (NSRF) 20072013. ARISTEIA-1893-ATLAS MICROMEGAS.

\section{REFERENCES}

[1] ATLAS Collaboration, JINST 3 S08003 (2008).

[2] ATLAS Collaboration, CERN-LHCC-2011-012, LHCC-I-020 (2011).

[3] ATLAS Collaboration, CERN-LHCC-2012-022, LHCC-I-023 (2012).

[4] ATLAS Collaboration, CERN-LHCC-2013-006, ATLAS-TDR-020 (2013).

[5] V. Smakhtin et al., Nucl. Instr. Meth. A 598 196-200 (2009).

[6] Y. Giomataris, et al. , Nucl. Instr. Meth. A376, 29-35, (1996).

[7] I. Giomataris, et al. , Nucl.Instrum.Meth. A560, 405-408, (2006).

[8] T. Alexopoulos et al., Nucl. Instr. Meth. A 640 110-118 (2011).

[9] G. Iakovidis, JINST 8 C12007 (2013).

[10] T. Alexopoulos et al., ATL-MUON-PUB-2013-002 (2013).

[11] Y. Kataoka et al., JINST 9 C03016 (2014).

[12] I. Giomataris et al. Nucl. Instr. Meth. A 560 405-408 (2006).

[13] M.J. French, et al., Nucl. Instr. Meth. A466, 359-365, (2001).

[14] S. Martoiu, et al., Nucl. Sc. Symp. and Med. Imag. Conf., IEEE, pp.2036,2038 (2011).

[15] G. De Geronimo, et al., IEEE Transactions on Nuclear Science, vol. 60, no. 3, (2013).

[16] M. Iodice, JINST 9 C01017 (2014).

[17] The ATLAS collaboration, ATL-UPGRADE-PUB-2014-001 (2014)

[18] R. Veenhof, Nucl. Instr. and Meth. A 419, 726, (1998).

[19] G. Iakovidis, CERN-THESIS-2014-148 (2014). 